\title{
Tetracycline Control
}

National Cancer Institute

\section{Source}

National Cancer Institute. Tetracycline Control. NCI Thesaurus. Code C20173.

In molecular biology, refers to the transcriptional regulation of a chimeric gene by the prokaryotic repressor/operator system of the tetracycline operon, permitting the production of a specific gene product or protein in a cell culture or a transgenic animal at controlled times. The chimeric gene contains the regulatory promoter elements of the tetracycline gene, and is transcriptionally regulated by the binding of recombinant tet repressor to these promoter elements in the absence of soluble tetracycline. 